\title{
Efeito Doppler gravitacional na órbita da estrela S2 em torno do buraco negro massivo Sgr A* no centro da Via Láctea
}

\author{
Gravitational Doppler effect in the orbit of the star S2 around the massive black hole Sgr A * at the center \\ of the Milky Way Galaxy
}

\author{
Wytler Cordeiro dos Santos ${ }^{*}[0$, Rafael dos Santos Magalhães 10 \\ ${ }^{1}$ Universidade de Brasília, 70910-900, Brasília, DF, Brasil.
}

\begin{abstract}
Recebido em 10 de agosto de 2020. Revisado em 11 de setembro de 2020. Aceito em 22 de setembro de 2020.
No ano de 2018 foi anunciado que a estrela S2, que vinha sendo monitorada desde 1992, orbitando o buraco negro massivo Sgr A* no centro da galáxia Via Láctea, obedecia as previsões da Teoria da Relatividade Geral de Einstein. Os movimentos orbitais dessa estrela e outras dezenas, em órbita do buraco negro Sgr A*, revelam que a massa do buraco negro é superior a quatro milhões de massas solares. A intensa gravidade do buraco negro Sgr A* causa um efeito Doppler gravitacional previsto na Teoria da Relatividade Geral e que foi observado na luz emitida pela estrela S2. Discutimos didaticamente os fenômenos envolvidos nessas observações realizadas para a estrela S2. Palavras-chave: Relatividade Geral, efeito Doppler, buraco negro massivo.
\end{abstract}

In 2018 it was announced that the star S2, which had been monitored since 1992, orbiting the massive black hole Sgr A * in the center of the Milky Way galaxy, obeyed as programmed by Einstein's Theory of General Relativity. The orbital movements of this star and dozens more in orbit of the black hole Sgr A * reveal that the mass of the black hole is greater than four million solar masses. The intense gravity of the black hole Sgr A * causes a gravitational Doppler effect predicted in the Theory of General Relativity and which was observed in the light emitted by the star S2. We didactically discuss the phenomena involved in these observations made for the star S2.

Keywords: General Relativity, Dppler efect, massive black hole.

\section{Introdução}

A Teoria da Relatividade Geral concebida por Albert Einstein em 1915, ao longo de mais de um século, se tornou a teoria mais bem sucedida da gravitação [1.2]. Historicamente pode-se destacar os fenômenos gravitacionais da precessão do periélio do planeta Mercúrio em órbita do Sol, o desvio de raios de luz das estrelas ao passarem próximos a campos gravitacionais fortes, como o desvio dos raios luminosos observados durante os eclipses totais do Sol. Várias previsões da Teoria da Relatividade Geral, como a geração de ondas gravitacionais por um sistema binário de pulsares provocando o decaimento no período orbital do sistema binário foram confirmadas. A primeira observação do decaimento no período orbital devido à radiação de ondas gravitacionais foi feita por Hulse e Taylor [3]. Eles usaram as observações precisas do período orbital do pulsar binário PSR B1913+16, que haviam descoberto em 1974, para calcular a radiação gravitacional emitida 44 7. Ondas gravitacionais previstas na Teoria da Relatividade Geral também foram detectadas diretamente pela primeira vez no observatório

*Endereço de correspondência: wytler@fis.unb.br. de detecção de ondas gravitacionai LIGO (Laser Interferometer Gravitational-Wave Observatory). A primeira observação de ondas gravitacionais foi registrada em setembro de 2015 [8] 9], que foram ondas geradas a partir da colisão entre dois buracos negros. Outra previsão importante da Teoria da Relatividade Geral foi a previsão da existência de buracos negros, regiões no espaço-tempo resultantes do colapso gravitacional de estrelas massivas, de onde a luz não pode escapar. A previsão teórica advém de resultados matemáticos da solução de Schwarzschild obtida em 1916 [5, 6, 10]. Duas importantes observações astrofísicas que revelam a existências de buracos negros massivos têm repercutido no meio científico recentemente: (i) a intensa fonte de radiação eletromagnética no objeto astrofísico denominado Virgo A localizado em M87 (Messier 87) 11 é provocada por um buraco negro supermassivo; (ii) Sgr A* (Sagittarius A*), também uma fonte intensa de radiação eletromagnética que está localizada no centro de nossa galáxia Via Láctea é a localização de um buraco negro massivo 12 .

Vamos fazer um estudo didático a respeito da aplicação da Teoria da Relatividade Geral de Einstein às descobertas recentes relacionadas ao efeito Doppler da luz 
emitida por uma das estrelas que orbitam muito próximo ao buraco negro massivo Sgr A* no centro da galáxia Via Láctea. A equipe GRAVITY (Max Planck Institute for extraterrestrial Physics, LESIA of Observatoire de $\mathrm{Pa}-$ ris/Université PSL/CNRS/Sorbonne Université/Université de Paris e IPAG da Université Grenoble Alpes/CNRS, the Max Planck Institute for Astronomy, University of Cologne, the CENTRA - Centro de Astrofisica e Gravitação, and the European Southern Observatory) [13], em 2018 divulgou os resultados observacionais/experimentais e confirmou mais uma vez as previsões teóricas da Relatividade Geral.

Dividimos didaticamente esse artigo em seis seções. $\mathrm{Na}$ seção II, aplicamos alguns dados astrométricos obtidos pelos grandes observatórios para mostrar didaticamente como obter a massa do buraco negro massivo Sgr A* e a velocidade orbital da estrela S2. Na seção III, discutimos didaticamente o efeito Doppler transverso. Na seção IV, abordamos o efeito Doppler gravitacional gerado por corpos massivos esféricos, usando a solução de Schwarzschild da Teoria da Relatividade Geral. Na seção V abordamos o efeito Rømer com um exemplo simples para órbitas circulares. A conclusão, na seção VI, finaliza a análise didática dos efeitos Doppler reunidos, explicando os resultados obtidos pela equipe científica que divulgaram os dados astrofísicos para a estrela S2.

\section{A órbita da estrela S2 em torno do buraco negro massivo Sgr $A^{*}$}

No centro da galáxia Via Láctea, existe uma fonte de raios $\mathrm{X}$, luz infravermelha e ondas de rádio muito intensa denominada de $\operatorname{Sgr} \mathrm{A}^{*}$ (Sagittarius A*) que é a localização de um buraco negro massivo, cuja massa é aproximadamente 4 milhões de vezes maior que a massa do Sol. Em órbita do buraco negro Sgr A*, existem dezenas de estrelas denominadas de S1, S2, S3, etc [14]. Algumas dessa órbitas são apresentadas no diagrama da Figura1

Uma delas se destaca por ser bem visível aos telescópios e ter a órbita bem determinada com período de 16,05 anos e semieixo maior em torno de 1.018,5 UA ( $1 \mathrm{UA}=1,5 \times 10^{11} \mathrm{~km}$ ) e excentricidade igual a aproximadamente 0.88466 13, 14. Medidas de astrometria foram cuidadosamente obtidas ao longo de 1992 a 2018, durante o período completo entre dois periápsides consecutivos (periápside: a menor distância entre a estrela S2 e Sgr $A^{*}$ ), principalmente pelas equipes do GRAVITY, NACO e SHARP 13. Um resumo dessa órbita é visto na Figura2

Mesmo orbitando um buraco negro massivo, é possível utilizarmos a gravitação clássica de Newton para verificarmos a massa do buraco negro Sgr A*. A estrela S2 tem um semieixo maior, $a=1.018,5 \mathrm{UA}$, e como veremos na seçao IV, o raio de Schwarzschild do buraco negro Sgr A*, é da ordem de 0,08 UA, de tal forma que a estrela orbita relativamente distante do centro de potencial

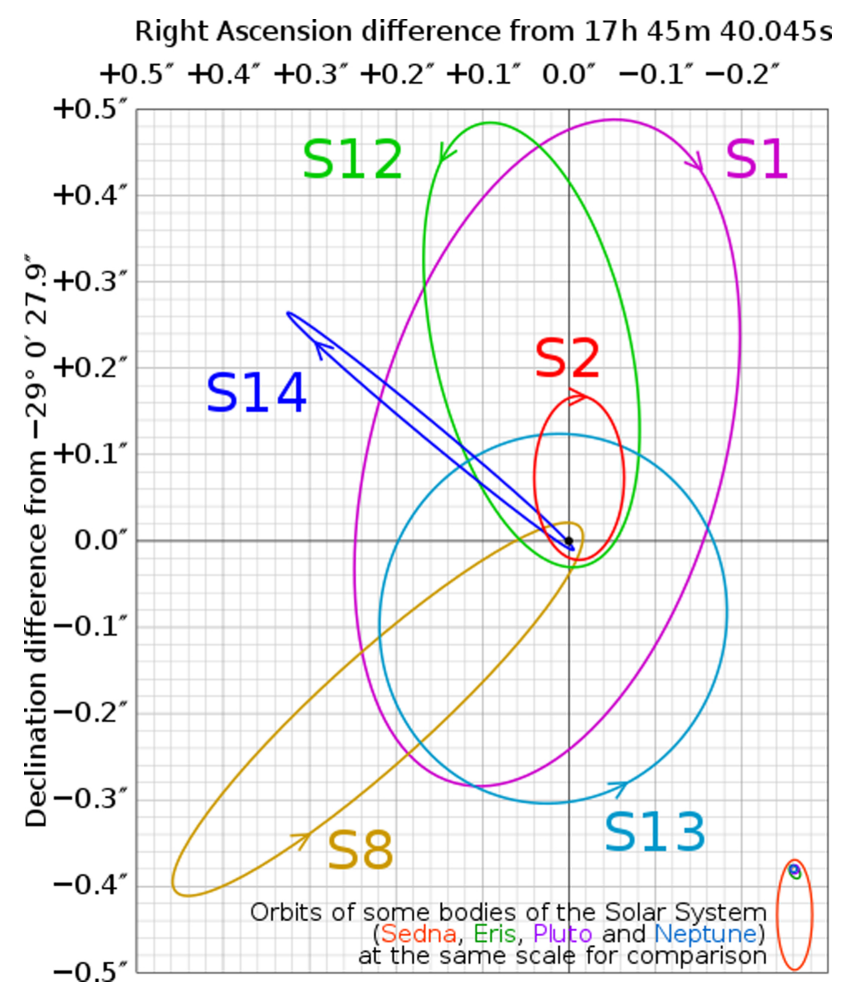

Figura 1: Órbita de seis estrelas em torno de Sgr A* 14. Os eixos referem-se às coordenadas celestes: ascensão reta $e$ declinação, que indicam a localização do buraco negro Sgr A* na esfera celeste [15].

gravitacional do buraco negro. O resultado obtido pela mecânica de Newton está muito próximo do resultado da Relatividade Geral, pois apesar da estrela S2 adquirir grandes velocidades no periápside para o níveis da Mecânica Clássica de Newton, em termos relativísticos, como veremos adiante, são relativamente pequenas. Então, de acordo com equação da órbita [16],

$$
r(\theta)=\frac{a\left(1-e^{2}\right)}{1+e \cos \theta},
$$

onde $r$ é a distância da estrela $\mathrm{S} 2$ ao buraco negro Sgr $\mathrm{A}^{*}, a$ é o semieixo maior da órbita, $e$ é a excentricidade da órbita elíptica e $\theta$ é a posição angular. Para $\theta=0$, obtemos a distância mais próxima entre os dois corpos, o periápside $r_{p}$,

$$
r_{p}=a(1-e),
$$

que para a órbita da estrela $\mathrm{S} 2$ resulta em $r_{p}=117,5$ UA.

Com a terceira lei de Kepler [17],

$$
\frac{T^{2}}{a^{3}}=\frac{M_{\odot}}{M_{\circledast}} \quad\left(\frac{\mathrm{anos}^{2}}{\mathrm{UA}^{3}}\right),
$$

onde $T$ é o período orbital da estrela $\mathrm{S} 2, T=16,05$ anos, $M_{\odot}$ é a massa do Sol e $M_{\circledast}$ é a massa do buraco negro, obtemos que a massa do $\mathrm{Sgr} \mathrm{A}^{*}$ é $M_{\circledast}=4,1 \times 10^{6} M_{\odot}$, ou seja, 4 milhões de massas solares [13]. 


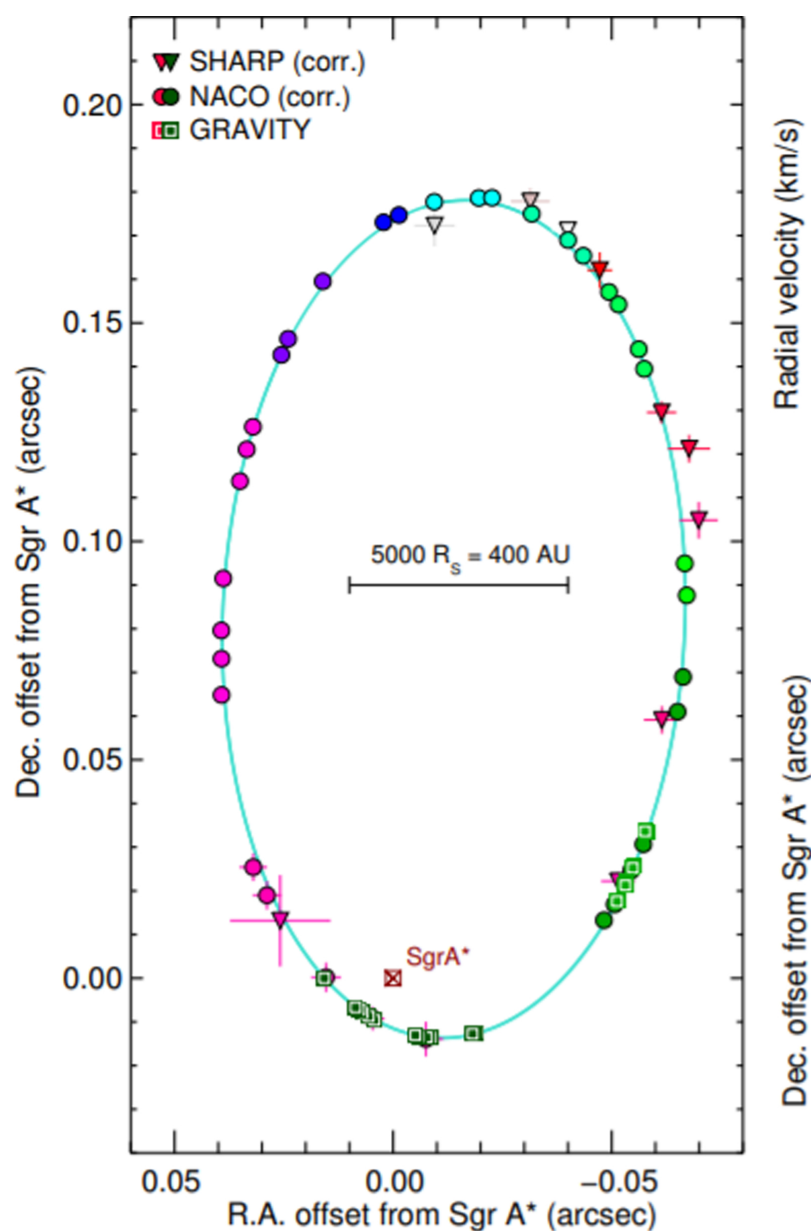

Figura 2: Sumário de um período orbital completo de S2 de 1992 até 2018 13.

Ainda no contexto da Mecânica Clássica de Newton, pode-se calcular a velocidade orbital da estrela S2 através da equação da energia do sistema de dois corpos: a estrela e o buraco negro Sgr $A^{*}$,

$$
E=\frac{1}{2} m v^{2}-\frac{G M_{\circledast} m}{r},
$$

onde $m \ll M_{\circledast}$ é a massa da estrela S2. Para uma órbita elíptica a energia total $E$ de um sistema de dois corpos é dada por,

$$
E=-\frac{G M_{\circledast} m}{2 a} .
$$

Substituindo o valor da energia total $E$ na equação (4) obtemos,

$$
m v^{2}=\frac{2 G M_{\circledast} m}{r}-\frac{G M_{\circledast} m}{a} .
$$

$\mathrm{Na}$ teoria da relatividade, o fator de Lorentz $\gamma=$ $\frac{1}{\sqrt{1-v^{2} / c^{2}}}$, é muito utilizado. Por isso, é conveniente, para os cálculos, divirdirmos a equação da velocidade orbital por $m c^{2}$, de forma que a equação acima se escreva como,

$$
\frac{v^{2}}{c^{2}}=\frac{2 G M_{\circledast}}{c^{2} r}-\frac{G M_{\circledast}}{c^{2} a} .
$$

Para cálculos posteriores usaremos a definição da razão entre a velocidade $v$ de um corpo e a velocidade da luz $c$, como $\beta=\frac{v}{c}$.

Com a equação (6), pode-se calcular a velocidade máxima que a estrela $\mathrm{S} 2$ adquire quando alcança o periápside em $r=r_{p}=117,5$ UA. Então, substituindo os valores numéricos obtidos anteriormente, podemos calcular a velocidade da estrela $\mathrm{S} 2$, que resulta em:

$$
v_{\max } \approx 0,0254 c \approx 7.620 \mathrm{~km} / \mathrm{s} .
$$

Observe que mesmo no ponto mais próximo entre a estrela S2 e o buraco negro Sgr $\mathrm{A}^{*}$, quando a velocidade da estrela é máxima, sua velocidade é 2,54\% da velocidade da luz. Isso implica que correções relativísticas devem ocorrer no cálculo do efeito Doppler da luz emitida pela estrela S2 devido a sua alta velocidade. Além de correções devido a curvatura do espaço-tempo gerada pela gravidade do buraco negro, que abordaremos nas próximas seções.

\section{Efeito Doppler Transversal}

Quando uma uma fonte luminosa se move em relação a um determinado observador, um sinal luminoso emitido pela fonte de comprimento de onda $\lambda_{0}$ ao ser captadado pelo observador é medido com comprimento de onda $\lambda_{1}<\lambda_{0}$, se a fonte se aproxima do observador, ou $\lambda_{1}>$ $\lambda_{0}$, se a fonte se afasta do observador 16. É útil fazermos uma discussão didática quando a fonte faz um ângulo $\theta$ entre a linha de deslocamento da fonte e a linha visagem, seguimento de reta que liga a fonte ao observador na origem $\mathcal{O}$. Para isso, consideremos uma fonte se movendo com velocidade $\boldsymbol{v}=v \boldsymbol{i}$ ao longo do eixo $x$ como é visto na Figura3.

Para verificar com exatidão o efeito Doppler observado pelo observador na origem $\mathcal{O}$, devemos projetar a componente da velocidade $\boldsymbol{v}$ da fonte ao longo da linha de visagem entre o observador e a fonte, que é o valor $v_{\|}=v \cos \theta$. Para a situação exemplificada na Figura 3 , o comprimento de onda detectado pelo observador é $\lambda_{1}<\lambda_{0}$, dado por,

$$
\lambda_{1}=(c-v \cos \theta) T
$$

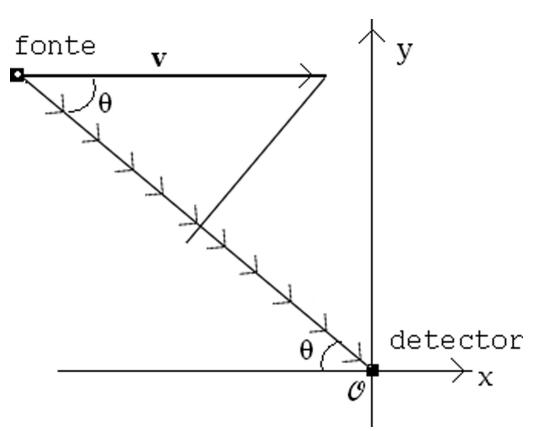

Figura 3: Efeito Doppler transversal. 
Onde $T$ é o período da onda luminosa detectado pelo observador. Se fizermos uma análise clássica newtoniana, em que as medidas de tempo do observador e da fonte são iguais, teremos que o intervalo de tempo da fonte $T_{0}=T$. Então a frequência da onda luminosa emitida pela fonte, $f_{0}=\frac{1}{T_{0}}=\frac{c}{\lambda_{0}}$ resulta para a equação 80 seguinte resultado,

$$
\lambda_{1}=\left(1-\frac{v}{c} \cos \theta\right) \frac{c}{f_{0}}=\left(1-\frac{v}{c} \cos \theta\right) \lambda_{0} .
$$

Para um efeito Doppler não levando em conta a teoria da relatividade, tem-se a seguinte equação para o desvio Doppler newtoniano,

$$
\begin{array}{ll}
\lambda_{1}-\lambda_{0}=-\lambda_{0} \frac{v}{c} \cos \theta & \text { fonte se aproxiamando, } \\
\lambda_{1}-\lambda_{0}=+\lambda_{0} \frac{v}{c} \cos \theta & \text { fonte se afastando. }
\end{array}
$$

Agora levando em conta a relatividade, o período $T$ é um tempo dilatado em relação ao período de tempo $T_{0}$ da fonte em movimento,

$$
T=\frac{T_{0}}{\sqrt{1-v^{2} / c^{2}}} .
$$

Então o comprimento de onda $\lambda_{1}$ na equação (8) detectado pelo observador passa a ser,

$$
\lambda_{1}=(c-v \cos \theta) \frac{T_{0}}{\sqrt{1-v^{2} / c^{2}}},
$$

vamos fazer uso da igualdade $T_{0}=\frac{\lambda_{0}}{c}$, que resulta para a equação acima,

$$
\lambda_{1}=\frac{\lambda_{0}}{c \sqrt{1-v^{2} / c^{2}}}(c-v \cos \theta),
$$

com a definição $\beta=\frac{v}{c}$ obtemos a equação do efeito Doppler,

$$
\frac{\lambda_{1}}{\lambda_{0}}=\frac{1-\beta \cos \theta}{\sqrt{1-\beta^{2}}} \quad \text { blueshift. }
$$

Essa equação é válida para o blueshift, desvio para o azul, quando o comprimento de onda captado é menor que o comprimento de onda emitido. Quando a fonte se afasta do observador, o sinal da velocidade se inverte $\boldsymbol{v} \rightarrow-\boldsymbol{v} \boldsymbol{i}$ e consequentemente a equação 10 passa a ser,

$$
\frac{\lambda_{1}}{\lambda_{0}}=\frac{1+\beta \cos \theta}{\sqrt{1-\beta^{2}}} \quad \text { redshift }
$$

a equação válida para o redshift, desvio para o vermelho, quando $\lambda_{1}>\lambda_{0}$, pois a fonte se afasta do observador.

Vamos definir o desvio Doppler como sendo

$$
\frac{\lambda_{1}-\lambda_{0}}{\lambda_{0}}=\frac{\lambda_{1}}{\lambda_{0}}-1=\beta_{D}
$$

Vamos considerar que a velocidade da fonte $v \ll c$ o que implica que $\beta^{2} \ll 1$ na equação 11 . Então pode-se fazer uma expansão em série de Taylor até a segunda ordem em $\beta$,

$$
\frac{\lambda_{1}}{\lambda_{0}}=(1+\beta \cos \theta)\left(1-\beta^{2}\right)^{-1 / 2} \approx 1+\beta \cos \theta+\frac{1}{2} \beta^{2},
$$

que se reescreve como

$$
\frac{\lambda_{1}}{\lambda_{0}}-1 \approx \beta \cos \theta+\frac{1}{2} \beta^{2}
$$

Usando a definição de desvio Doppler (12) podemos escrever a equação do desvio Doppler relativístico até a segunda ordem como:

$$
\beta_{D}=\beta \cos \theta+\frac{1}{2} \beta^{2} .
$$

Esse desvio Doppler é composto de um desvio Doppler clássico newtoniano, $\beta \cos \theta$, obtido na equação 9 mais um termo em segunda ordem, devido à teoria da relatividade especial.

Como vimos no resultado (7), a velocidade da estrela $\mathrm{S} 2$, em termos clássicos é uma velocidade alta, mas em termos relativísticos, é uma velocidade muito menor do que a velocidade da luz, o que torna a equação do desvio Doppler 13 útil para as aplicações na dinâmica orbital da estrela S2. Porém, não são somente esses valores da equação 13) que foram observados na dinâmica orbital da estrela S2. Conforme veremos na próxima seção, a curvatura do espaço-tempo, devido ao buraco negro Sgr $\mathrm{A}^{*}$, causa um desvio Doppler gravitacional que deve ser acrescentado ao desvio Doppler 13 .

\section{Efeito Doppler Gravitacional}

Na Teoria da Relatividade Geral, a primeira solução exata das equações de campo de Einstein foi a solução métrica de Schwarzschild, publicada por Karl Schwarzschild em 1916 18. Essa é a solução que descreve corpos astronômicos gravitantes esféricos com momento angular zero, mas que pode ser utilizada como aproximação para planetas e estrelas que giram em baixas velocidades. A solução de Schwarzschild é uma solução para o vácuo, para a região exterior à matéria da estrela ou planeta. Determinadas estrelas massivas que sofrem fortes colapsos gravitacionais podem ter a massa resultante do colapso gravitacional aprisionada em uma região do espaço-tempo em que a luz ou qualquer outro tipo de radiação não pode escapar dessa região. A solução de Schwarzschild descreve buracos negros estáticos (subentende-se com baixa velocidade angular), denominados de buracos negros de Schwarzschild, visto na equação abaixo,

$$
\begin{aligned}
d s^{2}= & \left(1-\frac{2 G M_{\circledast}}{c^{2} r}\right) c^{2} d t^{2}-\frac{d r^{2}}{\left(1-\frac{2 G M_{\circledast}}{c^{2} r}\right)} \\
& -r^{2}\left(d \theta^{2}+\sin ^{2} \theta d \phi^{2}\right) .
\end{aligned}
$$


Observe que quando $1-\frac{2 G M_{\circledast}}{c^{2} r}=0$, a métrica tem o setor temporal $c d t$ cancelado e o setor radial $d r$ divergente. $\mathrm{O}$ valor de $r$ em que ocorre essa singularidade, denotado por $r_{S}$ é dado por,

$$
r_{S}=\frac{2 G M_{\circledast}}{c^{2}} .
$$

Esse raio, conhecido como raio de Schwarzschild, é a distância radial que circunda a região onde a matéria se colapsou e de onde a radiação não pode escapar. O raio de Schwarzschild, também denominado de raio do buraco negro, é a região denominada de horizonte de eventos. Para o buraco negro Sgr A*, cuja massa foi calculada acima e vale $M_{\circledast}=4,1 \times 10^{6} M_{\odot}$, o raio de Schwarzschild é calculado pela equação (15) e o horizonte de eventos do buraco negro Sgr A* vale $r_{S}=1,21 \times 10^{7} \mathrm{~km}=0,08$ UA.

Vamos voltar na equação (6) onde temos,

$$
\beta^{2}=\frac{2 G M_{\circledast}}{c^{2} r}-\frac{G M_{\circledast}}{c^{2} a},
$$

a velocidade máxima que a estrela $\mathrm{S} 2$ pode adquirir é na posição do periápside $r_{p}=a(1-e)$. Usando a definição do raio de Schwarzschild (15) na equação $(16)$, obtemos para a velocidade ao quadrado máxima da estrela S2 a equação,

$$
\beta_{\max }^{2}=\frac{r_{S}(1+e)}{2 a(1-e)} .
$$

Vimos na seção II que o valor máximo para a estrela S2 é $\beta \approx 0,0254$.

Podemos definir um parâmetro relativístico 19,

$$
\Upsilon(r)=\frac{2 G M_{\circledast}}{c^{2} r},
$$

e ao comparar a equação acima com o raio de Schwarzschild da equação 15, podemos reescrever $\Upsilon(r)=\frac{r_{S}}{r}$. Com essa definição tem-se que $\Upsilon(a)=\frac{2 G M_{\circledast}}{c^{2} a}$, onde podese reescrever a equação 16 como,

$$
\beta^{2}=\Upsilon(r)-\frac{1}{2} \Upsilon(a)
$$

O efeito Doppler gravitacional, da mesma forma que o efeito Doppler cinemático discutido acima, é verificado entre uma fonte emissora de radiação eletromagnética em uma posição $r_{0}$ e um observador/receptor em uma posição $r_{1}$. Para a solução métrica de Schwarzschild o efeito Doppler gravitacional é dado pela equação [5],

$$
\frac{f_{1}}{f_{0}}=\frac{\left(1-\frac{2 G M_{\circledast}}{c^{2} r_{0}}\right)^{1 / 2}}{\left(1-\frac{2 G M_{\circledast}}{c^{2} r_{1}}\right)^{1 / 2}}
$$

onde $f_{0}$ é a frequência da radiação emitida, $f_{1}$ é a frequencia da radiação quando é captada por um observador. Em termos do comprimento de onda da radiação, a equação acima é reescrita como,

$$
\frac{\lambda_{0}}{\lambda_{1}}=\left(1-\frac{2 G M_{\circledast}}{c^{2} r_{0}}\right)^{1 / 2}\left(1-\frac{2 G M_{\circledast}}{c^{2} r_{1}}\right)^{-1 / 2} .
$$

Para o problema da estrela S2 em órbita do buraco negro Sgr $A^{*}$, a estrela S2 é a nossa fonte de radiação de comprimento de onda $\lambda_{0}$ e de posição variável $r_{0}=r$. Os observadores/receptores estão na Terra que captam uma radiação proviniente da estrela $\mathrm{S} 2$ de comprimento de onda $\lambda_{1}$ na posição $r_{1} \approx 26$ mil anos luz. Implica que o segundo termo do lado direito da equação (19) é o limite:

$$
\lim _{r_{1} \rightarrow \infty}\left(1-\frac{2 G M_{\circledast}}{c^{2} r_{1}}\right)=1 .
$$

Com esse resultado, a equação 19 se torna,

$$
\frac{\lambda_{0}}{\lambda_{1}}=\left(1-\frac{2 G M_{\circledast}}{c^{2} r}\right)^{1 / 2}
$$

e com a equação do raio de Schwarzschild 15 podemos expressar a equação do efeito Doppler gravitacional como,

$$
\frac{\lambda_{1}}{\lambda_{0}}=\left(1-\frac{r_{S}}{r}\right)^{-1 / 2}
$$

A estrela S2 no periápside, no ponto mais perto do buraco negro, tem $r_{p}=117,5 \mathrm{UA}$, e o raio de Schwarzschild, o horizonte de eventos, do buraco negro Sgr A* vale $r_{S}=0,08 \mathrm{UA}$, de forma que $\frac{r_{S}}{r} \ll 1$, e se torna possível fazer uma expansão de Taylor em primeira ordem na equação do efeito Doppler gravitacional acima,

$$
\frac{\lambda_{1}}{\lambda_{0}} \approx 1+\frac{r_{S}}{2 r}
$$

usando a definição $\Upsilon(r)=\frac{r_{S}}{r}$ essa equação resulta em

$$
\frac{\lambda_{1}}{\lambda_{0}}-1 \approx \frac{1}{2} \Upsilon(r)
$$

Definimos então o desvio Doppler gravitacional por $\beta_{G}=$ $\frac{\lambda_{1}}{\lambda_{0}}-1$ e a equação do desvio Doppler gravitacional se
escreve como,

$$
\beta_{G}=\frac{1}{2} \Upsilon(r) .
$$

Usamos agora a equação que dá a velocidade da estrela $\mathrm{S} 2$ em órbita do buraco negro, isolando $\Upsilon(r)$ na equação 18. e substituinda na equação 21, temos então,

$$
\beta_{G}=\frac{1}{2} \beta^{2}+\frac{1}{4} \Upsilon(a) .
$$

Entre os primeiros experimentos bem sucedidos que comprovam o desvio Doppler gravitacional, estão os experimentos de Pound \& Rebka em 1960 e depois melhorado por Pound \& Snider em 1965, que usaram o efeito Mossbauer para medir um desvio Doppler de raios gamas de 
$14.4 \mathrm{keV}$ emitidos por isótopos de ${ }^{57} \mathrm{Fe}$. O emissor e o detector dos raios gamas foram posicionados no topo e na base de uma torre de 22,5 metros. A medida de desvio Doppler gravitacional foi confirmada com apenas $1 \%$ de diferença entre o experimento e a Teoria da Relatividade Geral 20.

\section{Tempo de Atraso de Rømer}

O tempo de atraso de Rømer ou efeito Rømer é um efeito newtoniano causado pelo fato de que o tempo de viagem da luz emitida por um astro até o observador muda com as posições orbitais. Se uma estrela tem uma órbita em torno de um centro de massa cujo o plano da órbita tem uma inclinação em relação ao plano do céu, é possível medir um atraso de tempo da luz emitida pelo astro quando esse está mais distante do observador na Terra. Consideremos um exemplo hipotético de uma estrela em uma órbita circular em torno de um centro de massa, cujo o plano da órbita está totalmente perpendicular ao plano do céu, como visto na Figura 4 .

Consideremos que um observador faça uma observação da luz da estrela quando ela está na posição $P 1$, tal que luz terá viajado uma determinada distância até o observador na Terra. Quando a estrela for para a posição $P 2$ ela terá se afastado do observador e a luz emitida terá que percorrer uma distância maior até alcançar o observador na Terra. A diferença de tempo será $\delta t=\frac{d}{c}$, onde $d$ é a diferença espacial entre as posições $P 1$ e $P 2$ projetadas na linha de visagem entre a estrela e o observador na Terra. Observando a Figura 4 vemos que a distância $d$ é obtida por:

$$
d=R\left(\cos \theta_{2}-\cos \theta_{1}\right)
$$

para uma órbita circular o raio se mantem constante igual a $R$ e as mudanças de posições depende das variações angulares. Para a nossa proposta, vamos definir $\left(\cos \theta_{2}-\right.$ $\left.\cos \theta_{1}\right)=B_{R}$ um parâmetro para identificar o efeito Rømer nas equações do efeito Doppler.

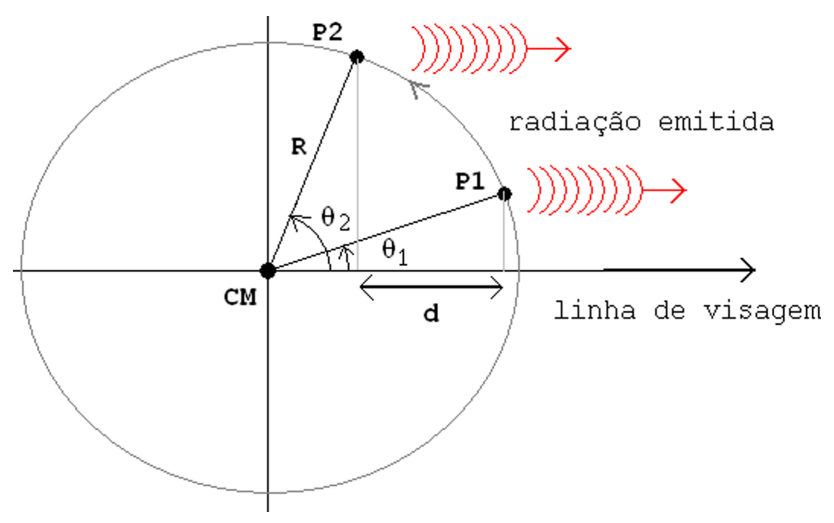

Figura 4: Tempo de atraso de Rømer. A linha de visagem conecta o centro de massa (CM) ao observador na Terra.
A velocidade radial $v_{r}$ da estrela medido na direção do observador na Terra será dado por $v_{r}=a_{r} \delta t$, sendo $a_{r}$ a aceleração da estrela na órbita circular,

$$
a_{r}=\frac{G M_{\circledast}}{R^{2}} .
$$

Podemos calcular a velocidade radial da estrela na direção de um observador na Terra,

$$
v_{r}=\frac{G M_{\circledast}}{R^{2}} \cdot \frac{B_{R} R}{c}=\frac{B_{R}}{c}\left(\frac{G M_{\circledast}}{R}\right) .
$$

Devemos observar da equação (5) que para uma órbita circular onde $r=a=R$, o quadrado da velocidade orbital da estrela é $v^{2}=\frac{G M_{\circledast}}{R}$. Então a equação acima pode ser simplificada,

$$
\frac{v_{r}}{c}=B_{R} \frac{v^{2}}{c^{2}} .
$$

O efeito Rømer contribui com $\beta^{2}=\frac{v^{2}}{c^{2}}$ ao efeito Doppler da luz emitida por uma estrela em movimento orbital. A equação acima então é reescrita $\operatorname{com} \beta_{R}=\frac{v_{r}}{c}$,

$$
\beta_{R}=B_{R} \beta^{2}
$$

Fizemos uma análise didática da contribuição que o tempo de atraso de Rømer dá ao efeito Doppler, assumindo uma órbita circular e com o plano orbital perpendicular ao plano do céu. Porém na maioria das observações reais, principalmente para os sistemas binários de estrelas, o plano orbital é inclinado em relação ao plano do céu e as órbitas são elípticas, o que torna o parâmetro $B_{R}$ difícil de ser medido e em geral o cálculo é feito computacionalmente $13,19,21]$. Deve-se observar que mesmo sendo uma contirbuição newtoniana ao efeito Doppler, a equação 23) participa no efeito Doppler com uma correção em $\beta^{2}$. Observe na Figura 2 que para cada ponto de observação, a mudança de posição da estrela S2 causa um tempo de atraso diferente de Rømer.

O nome do efeito do atraso de tempo entre sinais emitidos por estrelas é uma homenagem a Ole Rømer, astrônomo dinamarquês do final do século XVII, que foi o primeiro a observar esse efeito na lua de Júpiter, Io. A partir de diferentes posições orbitais da Terra, ele observou atrasos nos intervalos de ocultação da lua Io atrás de Júpiter, e dessa forma pode mensurar com aproximação a velocidade da luz 15$]$.

\section{Conclusão}

Fizemos uma análise dos principais fenômenos envolvendo o efeito Doppler que a luz emitida pela estrela S2 sofre na órbita em torno do buraco negro Sgr A*. Recapitulando os principais: efeito Doppler transversal, gravitacional 
e o efeito Rømer. Com os resultados $\sqrt{13}),(22)$ e $(23)$ escrevemos,

$$
\begin{aligned}
\beta_{z} & =\frac{\Delta \lambda}{\lambda}=\beta_{D}+\beta_{G}+\beta_{R} \\
& =\beta \cos \theta+\frac{1}{2} \beta^{2}+\frac{1}{2} \beta^{2}+\frac{1}{4} \Upsilon(a)+B_{R} \beta^{2} .
\end{aligned}
$$

Podemos simplificar a equação acima,

$$
\beta_{z}=B_{0}+B_{1} \beta+B_{2} \beta^{2} .
$$

O parâmetro $B_{0}$ engloba fatores independentes da velocidade $\beta$, incluindo aí o fator $\frac{1}{4} \Upsilon(a)$ que é devido ao potencial gravitacional do buraco negro. Além desse, pode ser acrescentada a composição do movimento do sistema solar em torno do centro galáctico, a energia potencial gravitacional de outras estrelas, etc. Porém, várias dessas contribuições ao efeito Doppler são pequenas e podem ser desprezadas. $\mathrm{O}$ fator $B 1=\cos \theta$ é a contribuição do efeito Doppler newtoniano. O fator $B_{2}=1+B_{R}$ é a soma dos efeitos Doppler da relatividade especial mais o efeito Doppler gravitacional e mais ainda o efeito Rømer.

A equipe GRAVITY 13 usou dados obtidos durante 26 anos, de 1992 até 2018, do monitoramento da velocidade radial da estrela $\mathrm{S} 2$. Os dados foram obtidos principalmente através de instrumentos do VLT-ESO: SINFONI e NACO, e de 2016 em diante, com a instrumentação do GRAVITY. Com os dados obtidos a equipe do GRAVITY anunciou que o efeito Doppler da luz emitida pela estrela S2 tinha a clara contribuiçao do efeito Doppler gravitacional (22). Com os dados obtidos e uma precisão instrumental (SINFONI, NACO e GRAVITY) para o efeito Doppler 24 de $\beta_{z} \approx 6,7 \times 10^{-4}$, a equipe GRAVITY usou vários métodos estatísticos e confirmou a previsão da Teoria da Relatividade Geral do efeito Doppler gravitacional, e afirmou que os dados obtidos para a luz emitida pela estrela S2 em órbita do buraco negro massivo Sgr A* seriam inconsistentes somente com a dinâmica de Newton.

\section{Agradecimentos}

Rafael dos Santos Magalhães reconhece e agradece o apoio e suporte financeiro recebido da Fundação de Apoio a Pesquisa do Distrito Federal - FAPDF.

\section{Referências}

[1] H. Weyl, Space, Time, Matter (Dover Publications, Mineola, 1952), $4^{\mathrm{a}}$ ed.

[2] S. Weinberg, Gravitation and Cosmology: Principles and Applications of the General Theory of Relativity (John Wiley \& Sons, Inc., New York, 1972).

[3] R.A. Hulse e J.H. Taylor, Astrophysical Journal 195, L51 (1975).

[4] J.M. Weisberg e J.H. Taylor, arXiv:astro-ph/0407149 (2005).
[5] M.P. Hobson, G.P. Efstathiou e A.N. Lasenby, General Relativity, an introduction for physicists (Cambridge University Press, Cambridge, 2006).

[6] B.F. Schutz, A First Course in General Relativity (Cambridge University Press, Cambridge, 2009), $2^{\mathrm{a}}$ ed.

[7] M.P. Ramos e R.V. Maluf, Revista Brasileira de Ensino de Física 40, e2302 (2018).

[8] https://www.ligo.caltech.edu/page/press-releasegw150914.

[9] M. Cattani e J.M.F. Bassalo, Revista Brasileira de Ensino de Física 38, e4202 (2016).

[10] R. D'Inverno, Introducing Einstein's Relativity (Oxford University Press, Clarendon, 1992).

[11] The Event Horizon Telescope Collaboration, The Astrophysical Journal Letters 875, L4 (2019).

[12] W.M. Goss, Robert L.Brown e K.Y. Lo, Astronomische Nachrichten 324, 497 (2003).

[13] GRAVITY Collaboration, R. Abuter, A. Amorim, M. Bauböck, J. P. Berger, H. Bonnet, W. Brandner, V. Cardoso, Y. Clénet, P.T. Zeeuw et al., Astronomy \& Astrophysics (A\&A) 615, L15 (2018).

[14] F. Eisenhauer, R. Genzel, T. Alexander, R. Abuter, T. Paumard, T. Ott, A. Gilbert, S. Gillessen, M. Horrobin, S. Trippe et. al., The Astrophysical Journal 628, 246 (2005).

[15] K.S. Oliveira Filho e M.F.O. Saraiva Astronomia e Astrofísica (Editora Livraria da Física, São Paulo, 2017), $4^{\mathrm{a}}$ ed.

[16] W. Greiner, Classical Mechanics, Point Particles and Relativity (Springer-Verlag, New York, 2004).

[17] R.G.G. Amorim e W.C. Santos, Revista Brasileira de Ensino de Física 39, e1310 (2017).

[18] A. Saa, Revista Brasileira de Ensino de Física 38, e4201 (2016).

[19] T. Alexander, Physics Reports 419, 65 (2005).

[20] C.W. Misner, K.S. Thorne e J.A. Wheeler, Gravitation (Princeton University Press, Princeton, 2017).

[21] S. Zucker, T. Alexander, S. Gillessen, F. Eisenhauer e R. Genzel, The Astrophysical Journal 639, L21 (2006). 